\title{
Analysis on Ecological Compensation of Wetland Protection in Western Sichuan
}

\author{
Zhang Dawei \\ Southwest Minzu University, Sichuan Chengdu, China
}

Keywords: western Sichuan; ethnic areas; wetland protection; sustainable development; strategy

\begin{abstract}
In the development of the western part of Sichuan Province in China, it is of great significance to strengthen the sustainable development of ecological wetlands in ethnic areas. Not only is it conducive to the promotion of further integration among ethnic groups, but it is also necessary for the realization of regional economic balance and stable development. In the establishment of the wetland industry in the ethnic areas of western Sichuan, it is necessary to adhere to the concept of sustainable development, that is, to integrate the regional culture of ethnic minorities with natural and ecological resources into a unified whole, to achieve national cultural balance, and to develop, use and protect the ecological environment. Under the premise of this, it is required to gradually build a sustainable wetland industrial pattern in the ethnic regions of western Sichuan.
\end{abstract}

\section{Introduction}

Sichuan Province is an important province in the western part of China and one of the major ethnic minorities. The geographical location of the western part of Sichuan Province is relatively remote and the terrain is more complex. The overall economic development level has lagged behind other regions. Western Sichuan is China's largest ethnically-inhabited area, with a large Tibetan population. The total population ranks second in all provinces across the country and is also the only ethnic group in China. It is this special atmosphere of multi-ethnic people living together and creating a unique atmosphere. However, due to the influence of various natural factors and ecological environment, the development of the wetland industry in the ethnic areas of western Sichuan has been severely constrained, and the contradiction between economic development and ecological protection has become increasingly fierce. This is also a key issue that must be resolved in the development of the wetland industry in the ethnic areas of western Sichuan.

\section{The Status Quo of Ecological Wetlands in Ethnic Areas of Western Sichuan}

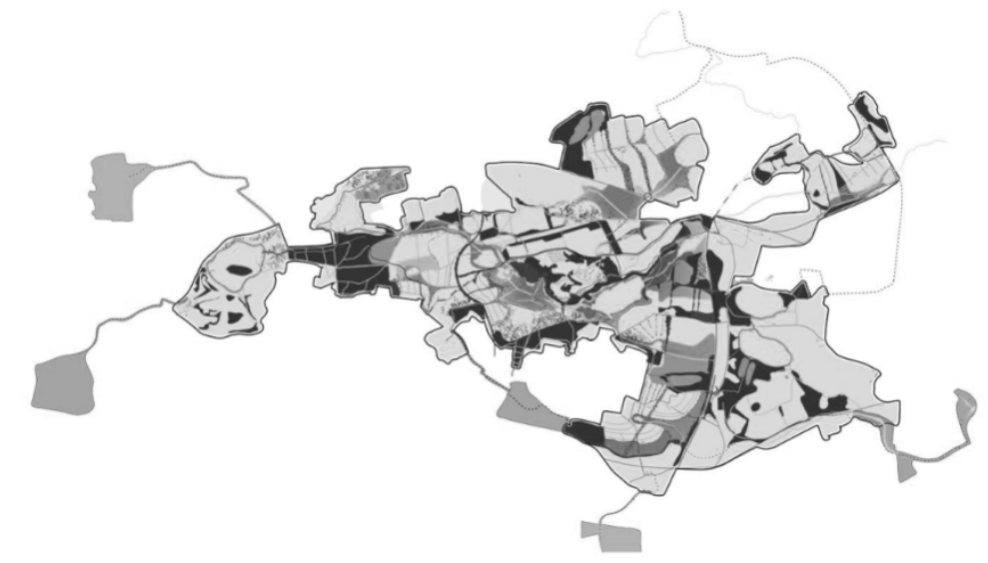

Fig.1 Seven well-established ecological compensation areas for wetland protection

In recent years, with the continuous development of our country's social economy and the continuous implementation of the western development policy, we have objectively promoted the development of the ecological wetland industry in the ethnic regions of western Sichuan, and has 
quickly become one of the pillar industries in the economic development of the ethnic regions in western Sichuan. In the development of China's modern wetland industry, the integration of material, ecological, and spiritual cultures is an inevitable development trend.

\section{Basic Principles for Sustainable Development of Ecological Wetlands in Ethnic Areas of Western Sichuan}

At present, in the economic development of the western Sichuan region, ecological wetlands in ethnic areas occupy an important position, but they are also facing increasingly severe economic and environmental conflicts. How to effectively realize the coordinated development and mutual promotion between them has become a national government in western Sichuan. In order to comply with the trend of socio-economic development, in the sustainable development of ecological wetlands in the ethnic areas of Western Sichuan, we must adhere to the following principles:

\subsection{Cultural Principles}

The establishment and development of the ecological wetland industry in the ethnic minority areas of western Sichuan is not only a new type of social economic activity, but also an important activity to promote the development of ethnic regional culture. In the sustainable development of ecological wetlands in the ethnic areas of western Sichuan, we must pay attention to cultural differences between regions and ethnic groups, and use the unique cultural heritage and charm of ethnic minorities to enable wetland tourists to truly experience and experience different from their own culture. In the development of China's modern wetland industry, the integration of material, ecological, and spiritual cultures is an inevitable development trend, and it is also an important cornerstone for adhering to cultural principles in the sustainable development of ecological wetlands in the ethnic minority areas of western Sichuan.

\subsection{Features Principle}

The so-called characteristic principle of the wetland industry mainly means that wetland areas must possess unique advantages that cannot be matched by their unique or other areas in terms of natural, ecological, and cultural resources, thereby fully demonstrating the nature of natural and human resources in wetland areas. . In the sustainable development of ecological wetlands in the ethnic areas of western Sichuan, through the development and use of excellent wetland resources of all ethnic groups, it is necessary to fully display the ecological, natural and cultural resources with local characteristics, especially the Tibetan, Buddhist and Buddhist communities living in this region.

\subsection{Market-oriented principles}

In the sustainable development of ecological wetlands in the ethnic areas of western Sichuan, the development, utilization, and protection of various types of ecological resources must adhere to the principle of market orientation. That is, the development of all ecological wetland projects must be based on market demand, and should be met to the utmost extent. On the basis of the consumption demand of wetland tourists, the construction and implementation of the ecological wetland protection system shall be strengthened. At the same time, we must reduce blindness and subjectivity in the development of ecological wetland projects in ethnic areas in western Sichuan, and try to achieve the matching of ecological wetland resources with the source markets, which is of great significance for promoting the long-term development of ethnic areas in western Sichuan.

\section{Strategies for Sustainable Development of Ecological Wetlands in Ethnic Areas of Western Sichuan}

In order to adapt to the new situation of China's social economy and the actual needs of regional wetland industry development, we must adhere to the concept of sustainable development in the construction of the ecological wetland industry in the ethnic areas of western Sichuan, which is to 
achieve the coordinated development of regional economic and social benefits. It is of great significance. In the sustainable development of ecological wetlands in the ethnic areas of western Sichuan, the key to solving is the contradiction between the economy, society and the environment, and the loss of the ecological environment caused by the development of wetland resources will be controlled within the minimum range.

\subsection{Straighten out relationships and strengthen coordination and management}

As far as the status quo of ecological wetland development in the western Sichuan region is concerned, most of the areas belong to the main functional area, but there are also a large number of restricted development areas, namely the ecological conservation areas demarcated by national or provincial governments. Combining with the status quo of ecological wetlands in the ethnic areas of western Sichuan, the sustainable development strategy mainly includes the following aspects:

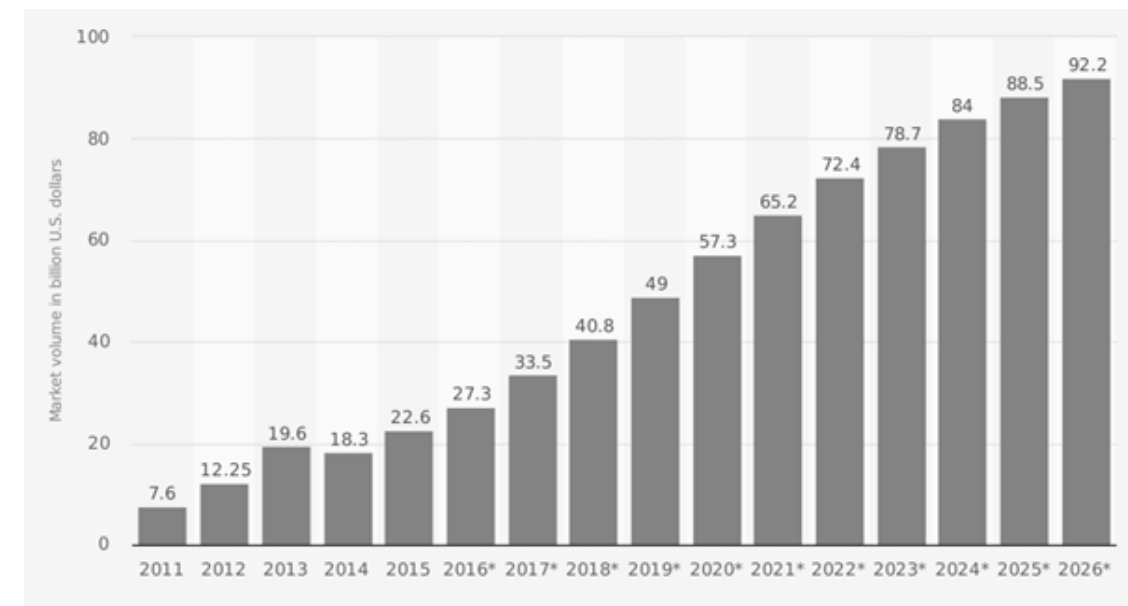

Fig.2 Construction and Development Forecast of Future Wetland Protected Areas

\subsection{Increase investment and improve the infrastructure construction in the ecological wetland area}

In order to further improve the quality of wetland services in the sustainable development of ecological wetlands in the ethnic areas of Western Sichuan, it is necessary to increase the investment and improve the infrastructure construction in the ecological wetland area. The total population ranks second in all provinces across the country and is also the only ethnic group in China. The economic benefits of developing and utilizing ecological resources to improve product quality are equal to the coefficient of interaction of ecological resources $(\alpha)$ multiplied by the product output of improved quality (Q) and multiplied by the product price (D2) and product quality (D1) before the increase in quality. The difference (D2-D1) is then subtracted from the cost (C).

$$
\mathrm{J}=\alpha \mathrm{Q}(\mathrm{D} 2-\mathrm{D} 1)-\mathrm{C}
$$

It is this special atmosphere of multi-ethnic people living together and creating a unique atmosphere.

\section{Summary}

In the sustainable development of ecological wetlands in the ethnic areas of western Sichuan, scientific, rational and effective measures must be taken. This has important implications for coordinating the internal and external demand structure in ethnic areas and enhancing the accumulation of capital in ethnic areas. Western ethnic areas must not only pay attention to their own superior resources, but also resolve the contradictions between nature, environment, and society. This has an irreplaceable role in promoting social civilization and economic development in the ethnic areas of western Sichuan, and it is also conducive to the promotion of ethnic integration. 


\section{References}

[1] Yang Z P. Analysis of the Ecological Compensation Based on Ecological Footprint Change in Elk Nature Reserve of Yancheng[J]. Research of Soil \& Water Conservation, 2011, 18(2):261-264.

[2] Wang L. Analysis of the Ecological Compensation Based on Ecological Footprint Change in

Red Crowned Crane Reserve of Yancheng[J]. Research of Soil \& Water Conservation, 2011, 18(3):272-251.

[3] Wang F X, Jiang L Q. ANALYSIS ON ECOLOGICAL COMPENSATION MECHANISM OF BIODIVERSITY PROTECTION [J]. Economic Geography, 2008. 